\title{
Effects of Total Knee Arthroplasty on Body Weight and Functional Outcome
}

\author{
BAYRAM UnVER, PT, PhD ${ }^{1)}$, VASFi KARATOSUn, MD ${ }^{2)}$, SERKAN BAKIRHAN, PT, PhD ${ }^{1)}$, \\ IZGE GUNAL, MD ${ }^{2}$ \\ ${ }^{1)}$ School of Physiotherapy, Dokuz Eylül University Hospital: Balcova, TR-35340, Izmir, \\ Turkey. TEL: +90 2322783118, FAX: +90 2322778180,E-mail: bayram.unver@deu.edu.tr \\ 2) Orthopedics, Dokuz Eylül University Hospital
}

\begin{abstract}
Purpose] We prospectively investigated the effects of arthroplasty surgery on body weight and the effects of obesity on functional outcomes and complications in patients with total knee arthroplasty (TKA). [Subjects] One hundred eighty-six TKA patients were divided into two groups [non-obese = body mass index $(B M I)<30 \mathrm{~kg} / \mathrm{m}^{2}(\mathrm{n}=70)$, and obese $\left.=B M I \geq 30 \mathrm{~kg} / \mathrm{m}^{2}(\mathrm{n}=116)\right]$ according to preoperative BMI values. [Methods] Patients were evaluated preoperatively and at 12 months postoperatively with regard to BMI, knee function score, pain, range of knee flexion, and complications. There were no significant postoperative differences between the groups in pain, knee function score, range of knee flexion, and complications.[Results] A statistically significant postoperative improvement was found in all parameters $(\mathrm{p}<0.05)$ except for BMI. In the non-obese group, BMI was unchanged whereas in the obese group there was an increase in BMI after surgery. [Conclusion] The results of this study indicate that patients do not lose weight after arthroplasty surgery. However, arthroplasty surgery offers significant improvements in knee flexion degree, knee function scores, physical function and relief of pain for patients regardless of weight. Increased mobility and decreased pain will not automatically lead to weight reduction. Therefore obesity must be addressed as an independent disease.
\end{abstract}

Key words: Total knee arthroplasty, Body weight, Functional outcome

(This article was submitted Nov. 5, 2008, and was accepted Feb. 19, 2009)

\section{INTRODUCTION}

Overweight (including obesity) is an established risk factor for arthritis in general and for osteoarthritis (OA) of weight-bearing joints such as the knee in particular ${ }^{1)}$. Weight reducing interventions must be considered as a first step in the management of knee pain in obese patients. Total knee arthroplasty (TKA) is a commonly performed procedure to treat OA of a knee which is unresponsive to conservative therapy ${ }^{2}$. Obesity has been considered an adverse influence on TKA outcomes by some authors and associated with increased peri-operative morbidity, including wound infections, prolonged hospital stay, and thromboembolism ${ }^{3,4)}$. Overweight patients are often advised against having TKA, or are warned that the results may be suboptimal ${ }^{3,5)}$. It has been estimated that a $10 \%$ reduction in body weight results in a $28 \%$ decline in knee symptoms and dysfunction in persons with debilitating $\mathrm{OA}^{6}$. When asked to lose weight preoperatively, for most patients, this requires starvation diets, leaving them malnourished at the time they enter the hospital. Lowered protein albumin levels render these patients at greater risk of infection ${ }^{7}$. Also, patients typically argue that joint pain and stiffness limit their mobility and thus their ability to use up calories $^{8)}$. They rationalize that losing weight would be easier postoperatively when the joint pain is 
relieved and the mechanical function restored ${ }^{9}$. However, three studies have opposed this rationalization $^{8-10)}$. These studies, focused only on the alterations of body weight, but not on the clinical outcomes such as postoperative function, pain, knee function score, range of motion and complications $^{8-10)}$. The importance of objective outcome measures in medicine has been realized in recent years ${ }^{3)}$, and we believe that a complete description of treatment outcome requires measures across all areas (ie: pain, range of motion, knee score, functional limitations, physical function and complications) of Nagi's model disablement ${ }^{11)}$.We aimed in this study to investigate the effects of arthroplasty surgery on body weight, and in addition to this, the effects of obesity on functional outcomes and complications.

\section{SUBJECTS AND METHODS}

A total of 186 patients (21 men and 165 women) with primary cemented TKA (58 unilateral, 128 bilateral TKA) performed by the same surgeon (VK) using the paramedian approach were included in this study. The preoperative diagnosis was OA for all patients, and only patients with primary total knee arthroplasty were included in this study. Patients with rheumatoid arthritis, septic arthritis, gout, paresis, metastatic bone disease or previous fracture of the lower limbs were excluded from the study. Patient height and weight and demographic data were recorded preoperatively and at 12 months postoperatively. Body Mass Index (BMI) was defined as weight in kilograms divided by the square of the patient's height in meters ${ }^{12)}$. Patients were stratified by obesity status using the preoperative BMI value. Non-obese subjects were defined as those with BMI values below $30 \mathrm{~kg} / \mathrm{m}^{2}$ and obese subjects were defined as those with BMI values above and equal to $30 \mathrm{~kg} / \mathrm{m}^{2}$. Thirty patients (non-obese $=8$ patients, obese $=22$ patients) had hypertension while 23 had diabetes mellitus (nonobese $=9$ patients, obese $=14$ patients). Mean patient age at surgery was $67.94 \pm 7.33$ years (range 40-85 years).

After surgery, all patients were allowed to bear weight as tolerated and physical therapy was started on the first postoperative day. All patients received standard postoperative treatment by a physiotherapist, including continuous passive motion, active-assisted and active range of motion exercises, isometric and isotonic strengthening exercises, gait training and transfer training ${ }^{13)}$. The patients were instructed to perform the exercises, and were postoperatively evaluated at 2, 4, 6, 12, 26 , and 52 weeks, in hospital, for examination and instruction of new exercises. The exercise program was taught to the participants by a physical therapist and performed in a home-based regimen. Patients were evaluated preoperatively, and at 12 months postoperatively with the parameters listed below.

The knee physical function of all patients was evaluated using the Hospital for Special Surgery (HSS) knee score criteria, which is based on a total of 100 points. The score is divided into the following categories: lack of pain (10 points); function (22 points); range of motion (18 points); muscle strength (10 points); flexion deformity (10 points); and lack of instability (30 points). Scores between 100 and 85 points were considered excellent results; scores between 84 and 70 were considered good; scores between 69 and 60 were considered fair; and scores below 60 were considered poor ${ }^{14}$. Knee active range of motion (ROM) was determined with a universal goniometer by evaluation of knee flexion ${ }^{15)}$. The postoperative course of each patient was reviewed for obesityrelated perioperative complications (complications related to the wound and thromboembolitic disease). The wound complications included necrosis of the skin, hematoma, and drainage from the wound.

\section{Statistical analyses}

The statistical analyses were performed using the independent samples $t$-test (age, weight, height, BMI, HSS score, pain score, functional activity score, ROM), paired $t$-test (weight, height, BMI, HSS score, pain score, functional activity score, ROM), and chi-square test (gender). A probability of $\mathrm{p}<0.05$ was considered to be significant.

\section{RESULTS}

The BMI distribution in the study population varied from a minimum of $26.42 \mathrm{~kg} / \mathrm{m}^{2}$ to a maximum of $39.90 \mathrm{~kg} / \mathrm{m}^{2}$, with a mean of 31.33 and $\mathrm{SD}$ of 4.02. According to preoperative BMI values, 70 patients were non-obese, 116 were obese.

None of the patients were lost to follow-up. The obese group had a few characteristics that were different from the non-obese group (Table 1, 
Table 1. Physical characteristics and results of evaluation parameters used in the study pre-surgery and post-surgery. $\Delta$ scores represent the change in value from pre-surgery to 12 months post-surgery

\begin{tabular}{|c|c|c|c|c|c|c|}
\hline & \multicolumn{2}{|l|}{ Pre-Surgery } & \multicolumn{2}{|c|}{1 year post-surgery } & \multicolumn{2}{|l|}{ Change $(\Delta)$} \\
\hline & Non-obese $(\mathrm{n}=70)$ & Obese $(n=116)$ & Non-obese $(n=70)$ & Obese $(n=116)$ & Non-obese $(n=70)$ & Obese $(n=116)$ \\
\hline $\operatorname{Age}(y)$ & $69.2 \pm 8.3$ & $67.1 \pm 6.5$ & & & & \\
\hline Sex (men/women) & $14 / 56$ & $7 / 109 *$ & & & & \\
\hline Weight (kg) & $69.8 \pm 9.0$ & $80.7 \pm 10.3^{*}$ & $69.9 \pm 8.6$ & $83.2 \pm 10.5^{*}$ & 0.1 & 2.4 \\
\hline Height $(\mathrm{cm})$ & $158.2 \pm 9.0$ & $155.6 \pm 6.8^{*}$ & $156.9 \pm 8.9$ & $154.3 \pm 6.3$ & -1.3 & -1.3 \\
\hline BMI $\left(\mathrm{kg} / \mathrm{m}^{2}\right)$ & $27.3 \pm 1.7$ & $33.7 \pm 2.9^{*}$ & $28.1 \pm 1.6$ & $34.8 \pm 3.5^{*}$ & 0.8 & 1.1 \\
\hline Pain Scores ${ }^{\mathrm{a}}$ & $13.7 \pm 8.9$ & $12.8 \pm 8.1$ & $25.7 \pm 3.8$ & $25.5 \pm 4.7$ & 12.7 & 12.0 \\
\hline Knee flexion degree $\left(^{\circ}\right)$ & $101.4 \pm 16.3$ & $96.9 \pm 17.9^{*}$ & $110.6 \pm 10.5$ & $110.4 \pm 9.7$ & 9.9 & 13.3 \\
\hline HSS Knee Score & $62.0 \pm 10.2$ & $60.3 \pm 11.9$ & $90.2 \pm 6.1$ & $89.2 \pm 8.7$ & 28.6 & 28.9 \\
\hline Walking Ability ${ }^{\mathrm{a}}$ & $5.9 \pm 2.6$ & $5.2 \pm 3.0^{*}$ & $10.5 \pm 1.6$ & $10.2 \pm 1.6$ & 4.6 & 5.1 \\
\hline Stair Climbing Ability ${ }^{\mathrm{a}}$ & $2.6 \pm 1.2$ & $2.2 \pm 0.7$ & $4.2 \pm 1.2$ & $4.0 \pm 1.3$ & 1.6 & 1.8 \\
\hline Transfer Ability ${ }^{\mathrm{a}}$ & $3.2 \pm 1.4$ & $2.8 \pm 1.3^{*}$ & $5.0 \pm 0.0$ & $5.0 \pm 0.0$ & 1.8 & 2.2 \\
\hline Functional Activity Score ${ }^{\mathrm{a}}$ & $11.7 \pm 3.8$ & $10.2 \pm 3.6^{*}$ & $19.7 \pm 1.9$ & $19.3 \pm 2.2$ & 8.0 & 9.1 \\
\hline
\end{tabular}

${ }^{*} \mathrm{p}<0.05$, HSS: Hospital for Special Surgery, BMI: Body Mass Index, ${ }^{\text {a }}$ Graded by HSS score.

$\mathrm{p}<0.05)$. There were no significant preoperative differences between the groups for evaluation parameters used in this study (pain scores, and HSS scores) except for knee flexion degree and functional activity score $(\mathrm{p}>0.05)$, (Table 1$)$. Preoperatively, the patients who were obese in this study had lower functional activity scores (10.2 points) than the patients who were non-obese (11.7 points $),(p=0.007)$, and the patients who were obese in this study had a lower range of knee flexion $\left(96.9^{\circ}\right)$ than the patients who were non-obese $\left(101.4^{\circ}\right),(\mathrm{p}=0.036)$.

There were no significant postoperative differences between the groups for the evaluation parameters used in the study (pain scores, knee flexion ROM, HSS score, and functional activity score) (Table 1). The obese patients, however, were heavier, with higher BMI scores than the non-obese group (Table 1), postoperatively. In both groups all parameters had improved at 12 months postoperatively compared with the preoperative values, except for the BMI values (Table 1).

When compared with the preoperative values, BMI of both groups increased postoperatively, however, this increase was significant only in the obese group (Table 1). The mean postoperative weight gain of the patients aged $\geq 65$ years was 3.34 $\mathrm{kg}$ (SD:5.74 kg, range: $-21 / 9, \mathrm{p}=0.000$ ) and $0.66 \mathrm{~kg}$ (SD:4.23 kg, range: $-8 / 9, p=0.281$ ) for the obese and non-obese groups, respectively. The mean postoperative weight gain of the patients aged $<65$ years was $1.20 \mathrm{~kg}$ (SD:7.39 kg, range:-20/24, $\mathrm{p}=0.260)$ and $-1.09 \mathrm{~kg}$ (SD:5.40 kg, range:-6/13, $\mathrm{p}=0.355$ ) for obese and non-obese groups, respectively. There was no correlation between age and functional results.

There were no surgically related complications in the non-obese group, but two obese patients experienced wound hemorrhage.

\section{DISCUSSION}

The effect of obesity on the outcome of TKA has been reported to be variable. Several reports have implicated excessive weight as a negative predictor of success in TKA ${ }^{1,16}$ ). Other authors have reported less significant differences in TKA outcomes between obese and nonobese patients ${ }^{4,17-20)}$. As stated in other studies, in our study there was no effect of obesity on functional outcomes (Table 1). Nevertheless, arthroplasty surgery does not result in weight loss. Overweight patients often claim their disabling joint disease limits their physical activity. They argue that an operation to eliminate their pain and to restore mechanical function will increase their activity levels and allow weight loss. Our results do not support this supposition. However, TKA offers significant improvement in knee ROM, knee function scores, physical function and relief of pain for patients regardless of weight. In our study, we found no difference between the non-obese and obese group patients according to the parameters of pain, ROM, knee score, and functional activity score $(\mathrm{p}>0.05)$.

There were some limitations of the study. Owing to the fact that morbid obese patients were not 
included in this study, the effect of arthroplasty on the body weight of this subgroup was not investigated. Also, it is hard to clarify and discuss in detail differences such as height and BMI alterations when a healthy control group is lacking. The activity status of the patients was measured using the functional activity score but pedometry only gives quantitative values. Thyroid hormone abnormalities are common in elderly patients. As body weight can be influenced by this, hormone blood levels should be assessed. In our study this was not done. Following arthroplasty obese patients should lose weight in order to maximize their functional status and extend the longevity of the implant, since as higher body weight leads to an overload on the components and surrounding bone tissue. A variety of dietetic interventions has been proven to be effective for weight loss. However, the effectiveness of these interventions depends on the supervision of the doctor and whether or not the patients follow their program. In our study, the diet of patients was not controlled.

The age-specific loss of height in different geographic locations and ethnic groups has been well documented. These studies show that height decreases with age ${ }^{21)}$. The cumulative change in height with aging will affect the interpretation of BMI, therefore BMI will increase "artifactually" as height is lost with aging ${ }^{21)}$. BMI, is often used as an index of obesity. It is a measure which is well suited for the purpose of determination of adiposity because it correlates closely with body mass, but only poorly with height, and it avoids the misleading conclusion of obesity based solely on weight without consideration of height $\left.{ }^{3}, 4\right)$. In our study, both obese and non-obese patients lost height over the 12-month period (Table 1). Our BMI change may partly be explained by this time dependent factor. Accordingly, in our opinion the increase in BMI was dependent not only on weight enhancement, but also on height lost. In the studies investigating the effects of arthroplasty on the weight $^{8-10)}$, patient height was not evaluated. These studies did not provide information about height, making it difficult to reach scientific conclusions and to make clinical recommendations. Consequently, in studies investigating the effect of arthroplasty surgery on obesity, in order to obtain objective outcomes, we think that not only body weight but also height needs to be evaluated.

Pain is a critical aspect of disability due to OA that can be resolved by surgery ${ }^{5}$. Obese patients argue that joint pain limits their mobility and their ability to use up calories ${ }^{8)}$. In spite of the pain complaints of the patients being reduced at one-year follow-up, there was no difference in the body weights (Table 1). Therefore we cannot say that weight loss can be assumed after the pain-reducing surgery. On the other hand, evidence suggests that diet-induced weight loss may be an effective method for improving knee symptoms and function while restricting the load bearing on the joint to normal activities ${ }^{6}$. A combination of physical exercise, behavior education and proper diet that focuses on weight reduction, healthier lifestyle and aerobic conditioning may enhance the ability of patients with TKA to perform daily activities. Since most daily activities are aerobic, obese patients would use up calories and eventually loose weight.

Obese patients had lower ROM both preoperatively $^{18)}$ and postoperatively ${ }^{22)}$. In the studies investigating the effects of arthroplasty on the weight ${ }^{8-10)}$, ROM was not evaluated. One of the most challenging goals following TKA surgery is regaining knee motion. Postoperative knee flexion should be sufficient to enable the patient to return to normal activities ${ }^{2)}$. Patients generally require at least 105 degrees of active knee flexion for basic daily activities ${ }^{2,23)}$. In several studies, knee flexion has been found to increase in the first year and potentially for up to 3 years ${ }^{23}$. The one-year followup mean knee flexion of our patients was $110^{\circ}$, which is higher than the suggested value and has the potential to increase further. In the obese patients, adequate joint ROM for activities of daily living was gained. Patients are encouraged to continue their exercises until the maximum benefit has been achieved.

It has been shown that obese TKA patients tend to be more sedentary and have lower activity levels ${ }^{17,24,25)}$. In the studies investigating the effects of arthroplasty on weight ${ }^{8-10)}$, activity level was not evaluated. Outcome evaluations of lower extremity joint reconstructions should include an assessment of patient activity ${ }^{26)}$. Walking and stair climbing have been identified by clinicians and patients as critical functional activities ${ }^{27}$ ). As such, they were used in this study to determine patient activity. The HSS score has a component representing functional activity with scores as high as 12 given for walking ability, as high as five for stair climbing ability, and as high as five for transfer ability for a total possible 
functional activity score of 22 . In the present study, when we evaluated the functional activity components of the HSS score in detail, the patients who were obese had lower functional activity scores (10.2 points) than the patients who were non-obese (11.7 points), $(\mathrm{p}=0.007)$, preoperatively. However, at the time of last assessment, the functional activity scores between the two groups were similar (obese $=19.3$, non-obese $=19.7, p=0.20$ ). TKA offers significant improvement in activity scores and function for patients regardless of weight. Many authors believe that a high body weight will lead to less-than-optimal TKA outcomes, because increased body weight leads to increased stress on the components and an increased load on the surrounding bone ${ }^{17,18)}$. Carrying extra weight adds stress to the knee in walking, running, climbing and descending stairs. Therefore, adequate phyical activity and exercise are essential for overweight patients. Caloric balance and weight control require regular physical activity ${ }^{28)}$. Additionally, cognitive behavioral therapy may be useful for weight control as reported by some studies of adolescents ${ }^{29,30)}$. These adjuncts should be used by obese patients to maximise the functional outcomes and the longevity of the implant.

Obese patients had a significantly lower preoperative total function score than non-obese patients 22,31$)$. However, in our study the postoperative scores of the groups were significantly better than at the preoperative stage (Table 1), and HSS scores were similar for the two groups. TKA offers significant improvement in knee scores and function for patients regardless of weight. We conclude that obese patients benefit greatly from TKA in the short term. Obesity alone should not be used as a reason to deny patients the benefit of this procedure. Additionally, after TKA, postoperative functional scores peaked at two years and subsequently declined ${ }^{32)}$. For this reason, we are thinking that patients should continue the rehabilitation program longer.

The results of this study indicate that patients do not lose weight after TKA with a 12-month followup. However, TKA offers significant improvement in knee ROM, knee function scores, physical function and relief of pain for patients regardless of their weight. The patients were expected lose weight after TKA. However, they were seen to put on weight, therefore obesity must be addressed as an independent disease and not rationalized as being dependent on physical inactivity due to joint disability. We conclude that non-obese and obese patients benefit greatly from TKA in the short term. However, studies with longer follow-up are needed to determine conclusively whether obesity is a predictor of a worse outcome after TKA.

\section{REFERENCES}

1) Mehrotra C, Naimi TS, Serdula M, et al.: Arthritis, body mass index, and professional advice to lose weight implications for clinical medicine and public health. Am J Prev Med, 2004, 27: 16-21.

2) Robinson RP, Simonian PT, McCann KJ: Rehabilitation following total knee arthroplasty. In: Fu FH, Harner CD, Vince KG (eds.), Knee surgery. Baltimore: Williams \& Wilkins, 1994, pp 1409-1425.

3) Deshmukh RG, Hayes JH, Pinder IM: Does body weight influence outcome after total knee arthroplasty? A 1-year analysis. J Arthroplasty, 2002, 17: 315-319.

4) Winiarsky R, Barth P, Lotke P: Total knee arthroplasty in morbidly obese patients. J Bone Joint Surg Am, 1998, 80: 1770-1774.

5) Hawker G, Wright J, Coyte P, et al.: Health-related quality of life after knee replacement. J Bone Joint Surg Am, 1998, 80: 163-173.

6) Christensen R, Astrup A, Bliddal H: Weight loss; the treatment of choice for knee osteoarthritis? A randomized trial. Osteoarthritis Cartilage, 2005, 13: 20-27.

7) Booth RE: Total knee arthroplasty in the obese patient. Tips and quips. J Arthroplasty, 2002, 17 (Suppl 1) : 69-70.

8) Woodruff MJ, Stone MH: Comparison of weight changes after total hip or knee arthroplasty. J Arthroplasty, 2001, 16: 22-24.

9) Heisel C, Silva M, Dela Rosa MA, et al.: The effects of lower-extremity total joint replacement for arthritis on obesity. Orthopedics, 2005, 28: 157-159.

10) Donovan J, Dingwall I, McChesney S: Weight change 1 year following total knee or hip arthroplasty. ANZ J Surg, 2006, 76: 222-225.

11) Nagi S: Disability concepts revisited: implications for prevention. In: Pope AM, Tarlov AR (eds.), Disability in America: Toward a National Agenda for Prevention. Washington: National Academy Pres, 1991, pp 309327.

12) Muir KR, Webber J: Weight loss and osteoarthritis. In: Brandt KD, Doherty M, Lohmander LS (eds.), Osteoarthritis. New York: Oxford University Press, 2003, pp 371-374.

13) Unver B, Karatosun V, Bakirhan S: Ability to rise independently from a chair during 6-month follow-up after unilateral and bilateral total knee replacement. J Rehabil Med, 2005, 37: 385-387.

14) Ranawat CS, Shine JJ: Duo-condylar knee 
arthroplasty. Clin Orthop Relat Res, 1973, 94: 185195.

15) Clarkson HM, Glewich GB: Musculoskeletal assessment, joint range of motion and manual muscle strength. Baltimore: Williams \& Wilkins, 1989.

16) Moreland JR: Mechanism of failure in total knee arthroplasty. Clin Orthop Relat Res, 1988, 226: 49-64.

17) Foran JR, Mont MA, Rajadhyaksha AD, et al.: Total knee arthroplasty in obese patients: a comparison with a matched control group. J Arthroplasty, 2004, 19: $817-824$

18) Griffin FM, Scuderi GR, Insall JN, et al.: Total knee arthroplasty in patients who were obese with 10 years followup. Clin Orthop Relat Res, 1998, 356: 23-28.

19) Smith BE, Askew MJ, Gradisar IA Jr, et al.: The effect of patient weight on the functional outcome of total knee arthroplasty. Clin Orthop Relat Res, 1992, 276: 237-244.

20) Unver B, Karatosun V, Bakirhan S: Effects of obesity on inpatient rehabilitation outcomes following total knee arthroplasty patients. Physiotherapy, 2008, 94: 198-203.

21) Sorkin JD, Muller DC, Andres R: Longitudinal change in the heights of men and women: consequential effects on body mass index. Epidemiol Rev, 1999, 21: 247-260.

22) Kjaersgaard-Andersen P, Hvid I, Wethelund JO, et al.: Total condylar knee arthroplasty in osteoarthritis. A four-to-six-year follow-up evaluation of 103 cases. Clin Orthop Relat Res, 1989, 238: 167-173.

23) Yashar AA, Colwell CW: Postoperative management and rehabilitation. In: Lotke PA, Garino JP (eds.),
Revision total knee arthroplasty. Philadelphia: Lippincott-Raven Publishers, 1999, pp 311-327.

24) Spicer DDM, Pomeroy DL, Badenhausen WE, et al.: Body mass index as a predictor of outcome in total knee replacement. Int Orthop, 2001, 25: 246-249.

25) McClung CD, Zahiri CA, Higa JK, et al.: Relationship between body mass index and activity in hip or knee arthroplasty patients. J Orthop Res, 2000, 18: 35-39.

26) Zahiri CA, Schmalzried TP, Szuszczewicz ES, et al.: Assessing activity in joint replacement patients. J Arthroplasty, 1998, 13: 890-898.

27) Walsh M, Woodhouse LJ, Thomas SG, et al.: Physical impairments and functional limitations: a comparison of individuals 1 year after total knee arthroplasty with control subjects. Phys Ther, 1998, 78: 248-258.

28) Minor MA: Physical therapy. In: Brandt KD, Doherty M, Lohmander LS (eds.), Osteoarthritis. New York: Oxford University Press, 2003, pp 299-305.

29) Tsiros MD, Sinn N, Brennan L, et al.: Cognitive behavioral therapy improves diet and body composition in overweight and obese adolescents. Am J Clin Nutr, 2008, 87: 1134-1140.

30) Lang A, Froelicher ES: Management of overweight and obesity in adults: Behavioral intervention for longterm weight loss and maintenance. Eur J Cardiovasc Nurs, 2006, 5: 102-114.

31) Stickles B, Phillips L, Brox WT, et al.: Defining the relationship between obesity and total joint arthroplasty. Obes Res, 2001, 9: 219-223.

32) Konig A, Scheidler M, Rader C, et al.: The need for a dual rating system in total knee arthroplasty. Clin Orthop Relat Res, 1997, 345: 161-167. 